\title{
Papers
}

\section{Midlife vascular risk factors and Alzheimer's disease in later life: longitudinal, population based study}

\author{
Miia Kivipelto, Eeva-Liisa Helkala, Mikko P Laakso, Tuomo Hänninen, Merja Hallikainen, \\ Kari Alhainen, Hilkka Soininen, Jaakko Tuomilehto, Aulikki Nissien
}

\begin{abstract}
Objective To examine the relation of midlife raised blood pressure and serum cholesterol concentrations to Alzheimer's disease in later life.

Design Prospective, population based study. Setting Populations of Kuopio and Joensuu, eastern Finland.

Participants Participants were derived from random, population based samples previously studied in a survey carried out in 1972, 1977, 1982, or 1987. After an average of 21 years' follow up, a total of 1449 (73\%) participants aged 65-79 took part in the re-examination in 1998.

Main outcome measures Midlife blood pressure and cholesterol concentrations and development of Alzheimer's disease in later life.

Results People with raised systolic blood pressure $(\geqslant 160 \mathrm{~mm} \mathrm{Hg})$ or high serum cholesterol concentration ( $\geqslant 6.5 \mathrm{mmol} / \mathrm{l})$ in midlife had a significantly higher risk of Alzheimer's disease in later life, even after adjustment for age, body mass index, education, vascular events, smoking status, and alcohol consumption, than those with normal systolic blood pressure (odds ratio $2.3,95 \%$ confidence interval 1.0 to 5.5) or serum cholesterol (odds ratio 2.1, 1.0 to 4.4). Participants with both of these risk factors in midlife had a significantly higher risk of developing Alzheimer's disease than those with either of the risk factors alone (odds ratio 3.5, 1.6 to 7.9). Diastolic blood pressure in midlife had no significant effect on the risk of Alzheimer's disease.

Conclusion Raised systolic blood pressure and high serum cholesterol concentration, and in particular the combination of these risks, in midlife increase the risk of Alzheimer's disease in later life.
\end{abstract}

\section{Introduction}

Risk factors for vascular disease may also be risk factors for Alzheimer's disease. Some cross sectional studies have investigated the relation of blood pressure and cholesterol concentration to cognitive function in late life, but with conflicting results. Furthermore, one limitation of cross sectional studies is that they cannot readily determine causality. This shortcoming can to some extent be overcome with longitudinal, population based studies. Two such studies have found that raised blood pressure precedes the development of Alzheimer's disease-one conducted in a Swedish population aged 70 years and followed for 9-15 years and the other in Japanese-American men with a mean age of 53 followed for 25 years. ${ }^{12}$ In addition, Finnish men aged 70-89 with Alzheimer's disease were found to have had raised serum cholesterol concentrations 15 -25 years before the onset of the disease. ${ }^{3}$

It is important to identify early risk factors for Alzheimer's disease because the neurodegenerative processes of Alzheimer's disease may begin in midlife. ${ }^{4}$ Identification of these risk factors may shed some light on the pathophysiology of Alzheimer's disease and also provide new potential avenues for its prevention and treatment. The preliminary findings showing an association between vascular risk factors and Alzheimer's disease need to be replicated in independent populations, and no population based study has yet evaluated the association of both midlife blood pressure and cholesterol concentrations with Alzheimer's disease in later life in both sexes. We investigated the putative impact of raised blood pressure and cholesterol concentrations in midlife on the subsequent development of Alzheimer's disease in a population based sample.

\section{Participants and methods}

Participants were derived from four separate independent population based samples studied within the framework of the North Karelia project and the FINMONICA study in 1972, 1977, 1982, and 1987.5 Participants who were still alive, aged $65-79$ by the end of 1997, and living in two geographically defined areas in or close to the towns of Kuopio and Joensuu were the target of this study. From these 2293 people, a random sample of 2000 participants were invited to the re-examination carried out during 1998. Altogether $1449(72.5 \%)$ people participated (figure on website). The mean length of follow up was 21 (SD 4.9) years (range 11-26 years; 26 years for 34.9\%, 21 years for $38.1 \%, 16$ years for $15.6 \%$, and 11 years for $11.4 \%$ of participants). The study was approved by the local ethics committee, and written informed consent was obtained from all participants.

\section{Examination and re-examination}

The protocol for the midlife examination is described in greater detail elsewhere. ${ }^{5}$ In brief, the survey

\section{Department of Neuroscience and Neurology, University of Kuopio, PO Box 1627,70211 Kuopio, Finland Miia Kivipelto research fellow Merja Hallikainen research fellow \\ Department of Public Health and General Practice, University of Kuopio \\ Eeva-Liisa Helkala neuropsychologist Aulikki Nissinen professor \\ Department of Clinical Radiology, Kuopio University Hospital, PO Box 1777, 70211, Kuopio, Finland Mikko P Laakso research fellow, Academy of Finland \\ Department of Neurology, Kuopio University Hospital \\ Tuomo Hänninen neuropsychologist Hilkka Soininen professor}

continued over

BMJ 2001;322:1447-51

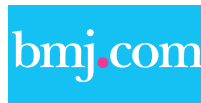

Figure showing the study design appears on the BMJ's website 
North Karelia Central Hospital, 80210 Joensuu,

Finland

Kari Alhainen

department head

physician

National Public Health Institute, 00300 Helsinki, Finland

Jaakko Tuomilehto professor

Correspondence to: M Kivipelto miia.kivipelto@uku.fi

Table 1 Sociodemographic and clinical characteristics of the study population. Values are means (standard deviations) unless otherwise stated

\begin{tabular}{|c|c|c|c|c|c|c|}
\hline Characteristic & $\begin{array}{l}\text { Alzheimer's disease } \\
\qquad(\mathrm{n}=48)\end{array}$ & $\begin{array}{l}\text { No dementia } \\
(\mathrm{n}=1352)\end{array}$ & $P$ value & $\begin{array}{l}\text { Participants } \\
(n=1409)\end{array}$ & $\begin{array}{l}\text { Non-participants } \\
(\mathrm{n}=591)\end{array}$ & $\begin{array}{l}\text { P value } \\
(t \text { test })\end{array}$ \\
\hline Male/female (\%) & $40 / 60$ & $38 / 62$ & $0.73^{\star}$ & $38 / 62$ & $36 / 64$ & $0.57^{*}$ \\
\hline Duration of follow up (years) & $20.6(4.5)$ & $20.9(4.9)$ & 0.75 & $20.9(4.9)$ & & \\
\hline \multicolumn{7}{|l|}{ Age (years): } \\
\hline Midlife & $54.0(4.7)$ & $50.2(6.0)$ & $<0.001$ & $50.4(6.0)$ & $51.2(5.9)$ & 0.007 \\
\hline Re-examination & $74.7(3.8)$ & $71.1(4.0)$ & $<0.001$ & $71.3(4.0)$ & & \\
\hline Education (years) & $6.7(2.7)$ & $8.5(3.5)$ & $<0.001$ & $8.4(3.5)$ & $7.4(2.9)$ & $<0.001$ \\
\hline Mini-mental state examination & $20.4(3.0)$ & $26.1(2.0)$ & $<0.001$ & $25.9(2.4)$ & & \\
\hline \multicolumn{7}{|l|}{ Body mass index $\left(\mathrm{kg} / \mathrm{m}^{2}\right)$ : } \\
\hline Midlife & $27.6(4.0)$ & $26.5(3.7)$ & 0.038 & $26.6(3.7)$ & $27.2(4.5)$ & 0.002 \\
\hline Re-examination & $27.8(4.4)$ & $27.8(4.3)$ & 0.994 & $27.8(4.3)$ & & \\
\hline \multicolumn{7}{|l|}{ Systolic blood pressure $(\mathrm{mm} \mathrm{Hg})$ : } \\
\hline Midlife & $152.7(18.2)$ & $143.9(19.8)$ & 0.002 & $144.3(19.9)$ & $150.2(21.7)$ & $<0.001$ \\
\hline Re-examination & $150.4(23.5)$ & $151.5(23.4)$ & 0.747 & $151.4(23.3)$ & & \\
\hline \multicolumn{7}{|c|}{ Diastolic blood pressure $(\mathrm{mm} \mathrm{Hg})$ : } \\
\hline Midlife & $91.2(9.5)$ & $89.1(10.9)$ & 0.182 & $89.2(10.9)$ & $91.7(11.3)$ & $<0.001$ \\
\hline Re-examination & $80.4(11.5)$ & $80.5(11.2)$ & 0.965 & $80.4(11.2)$ & & \\
\hline \multicolumn{7}{|l|}{ Cholesterol $(\mathrm{mmol} / \mathrm{l})$ : } \\
\hline Midlife & $7.2(1.0)$ & $6.7(1.2)$ & 0.001 & $6.7(1.2)$ & $7.0(1.3)$ & $<0.001$ \\
\hline Re-examination & $6.0(1.0)$ & $5.8(1.0)$ & 0.234 & $5.8(1.0)$ & & \\
\hline
\end{tabular}

${ }^{*} \chi^{2}$ test was used.

included a self administered questionnaire on medical history, cerebrovascular and cardiovascular events, and vascular conditions diagnosed by a physician. Height and weight were measured. Blood pressure was measured in the right arm after participants had been seated for five minutes. Venous blood specimens were taken for determination of serum cholesterol concentrations. $^{5}$

During the re-examination in 1998, the survey methods followed those of the previous surveys in all aspects. Dementia was diagnosed in a three phase study design: a screening phase (phase 1), a clinical phase (phase 2), and a differential diagnosis phase (phase 3) (figure on website). The diagnosis of dementia was based on the Diagnostic and Statistical Manual of Mental Disorders, fourth edition, ${ }^{6}$ and the diagnosis of Alzheimer's disease was made according to the criteria of the National Institute of Neurological and Communicative Disorders and Stroke and the Alzheimer Disease and Related Disorders Association.?

A total of $57(4 \%)$ participants were diagnosed as having dementia, of whom 48 fulfilled the diagnostic criteria for probable or possible Alzheimer's disease. All patients with Alzheimer's disease showed generalised or medial temporal atrophy, and none showed appreciable vascular pathology on magnetic resonance scans. Isolated minor lacunae or periventricular white matter signal changes were not considered as exclusion criteria for Alzheimer's disease. All patients with Alzheimer's disease scored four or less on the Hachinski ischaemia scale. ${ }^{8}$

Four patients were diagnosed as having vascular dementia, two as having Parkinson's disease with dementia, two as having alcoholic dementia, and one as having frontotemporal dementia. Since the focus of this study was Alzheimer's disease and the number of participants with non-Alzheimer dementia was small, these nine cases were excluded from further analyses.

\section{Statistical analyses}

Differences between the study groups were analysed with Student's $t$ test and the $\chi^{2}$ test as appropriate.
Midlife blood pressure values were classified into normal $(<140 \mathrm{~mm} \mathrm{Hg}$ ), borderline (140-159 $\mathrm{mm} \mathrm{Hg}$ ), and high $(\geqslant 160 \mathrm{~mm} \mathrm{Hg})$ for systolic pressure; and normal $(<90$ $\mathrm{mm} \mathrm{Hg}$ ), borderline (90-94 $\mathrm{mm} \mathrm{Hg}$ ), and high ( $\geqslant 95 \mathrm{~mm}$ $\mathrm{Hg}$ ) for diastolic pressure. Midlife serum cholesterol concentrations were classified into high $(\geqslant 6.5 \mathrm{mmol} / \mathrm{l})$ and normal $(<6.5 \mathrm{mmol} / \mathrm{l})$. The association between midlife blood pressure and cholesterol concentration and subsequent Alzheimer's disease was investigated with multiple logistic regression analyses, using normal blood pressure and cholesterol as the reference category (model 1). As blood pressure and cholesterol concentration are influenced by age and body mass index, the analyses were replicated controlling for these factors (model 2). Further analyses were carried out controlling also for education, history of myocardial infarction and cerebrovascular symptoms (used as indicators of vascular events), and smoking status and alcohol consumption (model 3).

\section{Results}

\section{Sociodemographic and clinical characteristics}

Table 1 shows the sociodemographic and clinical characteristics of the study population. Mean age was 50.4 years (SD 6.0, range 40-64 years) in the original survey and 71.3 years $(4.0,65-80)$ at re-examination. Patients with Alzheimer's disease were significantly older, and they also had less formal education than participants without dementia. Patients with Alzheimer's disease had higher body mass index, systolic blood pressure, and serum total cholesterol in midlife than did participants without dementia, but at re-examination these values were similar in the two groups. Diastolic blood pressure was similar in patients with Alzheimer's disease and participants without dementia both in midlife and at re-examination.

\section{Blood pressure, cholesterol, and risk of Alzheimer's disease}

High systolic blood pressure in midlife was a significant risk for Alzheimer's disease in later life (table 2), and 
Table 2 Association of midlife blood pressure and cholesterol concentrations with Alzheimer's disease later in life. Values are numbers (percentages) unless otherwise stated

\begin{tabular}{|c|c|c|c|c|c|}
\hline \multirow[b]{2}{*}{ Characteristic } & \multirow{2}{*}{$\begin{array}{l}\text { Alzheimer's disease } \\
\qquad(\mathrm{n}=48)\end{array}$} & \multirow{2}{*}{$\begin{array}{l}\text { No dementia } \\
(n=1352)\end{array}$} & \multicolumn{3}{|c|}{ Odds ratio $(95 \% \mathrm{Cl})$} \\
\hline & & & Model $1^{*}$ & Model 2* & Model 3* \\
\hline \multicolumn{6}{|c|}{ Systolic blood pressure $(\mathrm{mm} \mathrm{Hg})$ : } \\
\hline$<140$ (normal) & $12(25)$ & $606(44.8)$ & 1.0 & 1.0 & 1.0 \\
\hline 140-159 (borderline) & $20(42)$ & $484(35.8)$ & $2.1(1.0$ to 4.3$)$ & 1.8 (0.85 to 3.7$)$ & $2.1(0.8$ to 5.0$)$ \\
\hline$\geqslant 160$ (high) & $16(33)$ & $262(19.4)$ & $3.1(1.4$ to 6.6$)$ & $2.3(1.0$ to 5.0$)$ & 2.8 (1.1 to 7.2$)$ \\
\hline \multicolumn{6}{|c|}{ Diastolic blood pressure $(\mathrm{mm} \mathrm{Hg})$ : } \\
\hline$<90$ (normal) & $21(44)$ & $704(52.1)$ & 1.0 & 1.0 & 1.0 \\
\hline 90-94 (borderline) & $9(19)$ & $289(21.4)$ & $1.0(0.47$ to 2.3$)$ & 1.1 (0.48 to 2.4$)$ & $1.4(0.6$ to 3.5$)$ \\
\hline$\geqslant 95$ (high) & $18(38)$ & $359(26.6)$ & $1.7(0.88$ to 3.2$)$ & $1.6(0.84$ to 3.2$)$ & 1.7 (0.8 to 3.6$)$ \\
\hline \multicolumn{6}{|c|}{ Cholesterol concentration $(\mathrm{mmol} / \mathrm{l})$ : } \\
\hline$<6.5$ (normal) & $11(23)$ & $630(46.6)$ & 1.0 & 1.0 & 1.0 \\
\hline$\geqslant 6.5$ (high) & $37(77)$ & $722(53.4)$ & $2.9(1.5$ to 5.8$)$ & $2.7(1.4$ to 5.4$)$ & $2.2(1.0$ to 4.7$)$ \\
\hline \multicolumn{6}{|l|}{ Risk groups I: $†$} \\
\hline Single risk factor & $23(48)$ & $660(48.8)$ & 1.0 & 1.0 & 1.0 \\
\hline Combined & $15(31)$ & $162(12.0)$ & 2.7 (1.4 to 4.4$)$ & 2.2 (1.1 to 4.4$)$ & 3.5 (1.6 to 7.9$)$ \\
\hline \multicolumn{6}{|l|}{ Risk groups II: $†$} \\
\hline Single risk factor & $13(27)$ & $614(45.4)$ & 1.0 & 1.0 & 1.0 \\
\hline Combined & $30(63)$ & 427 (31.6) & $3.3(1.7$ to 6.4$)$ & 2.9 (1.5 to 5.7$)$ & 2.8 (1.3 to 5.9) \\
\hline
\end{tabular}

*Model 1 gives univariate odds ratios for the risk of Alzheimer's disease; model 2 controls for age and body mass index; model 3 controls for age, body mass index, education, history of myocardial infarction and cerebrovascular symptoms, smoking status, and alcohol consumption.

†In risk groups I, single risk factor indicates either systolic blood pressure $\geqslant 160 \mathrm{~mm} \mathrm{Hg}$ or cholesterol $\geqslant 6.5 \mathrm{mmol} / \mathrm{l}$, and combined indicates both of these risk

factors. Risk groups II were similar, but the cut-off point for systolic blood pressure was $140 \mathrm{~mm} \mathrm{Hg}$.

this remained true after all the adjustments (models 2 and 3). Borderline high systolic blood pressure in midlife also increased the risk (model 1), but after adjustments (models 2 and 3 ) this association was no longer significant. Midlife diastolic blood pressure had no significant effect on the risk of Alzheimer's disease in any of the models. High serum cholesterol concentration in midlife was a significant risk for Alzheimer's disease, even after all the adjustments (models 1-3).

Because high systolic blood pressure and cholesterol concentration were significant risk factors for Alzheimer's disease, we evaluated the added risk related to possessing both these risk factors. Participants with both risk factors in midlife had significantly higher risk for Alzheimer's disease than did those with either of these risk factors alone (table 2).

Analyses included control for age at the time when risk factor data were collected, because the effects of the midlife variables were the focus of this study. The length of follow up did not differ between participants who developed Alzheimer's disease and those who did not; thus controlling for age at re-examination did not change the results (data not shown).

\section{Medical history and Alzheimer's disease}

Table 3 shows the medical history of the participants. Patients with Alzheimer's disease were more likely to have been treated with antihypertensive drugs in midlife, but at re-examination later in life there was no difference between the groups. At re-examination, patients with Alzheimer's disease were significantly more likely to have a history of myocardial infarction and cerebrovascular symptoms (almost invariably expressed as transient ischaemic attack) and less likely to be alcohol users than participants without dementia.

\section{Discussion}

This study showed that raised systolic blood pressure and high serum cholesterol concentrations in midlife increased the risk of Alzheimer's disease in later life.
The combination of these risk factors in midlife, even when participants with borderline high systolic blood pressure were included, increased the risk to a greater extent than either of the risk factors on its own. Diastolic blood pressure in midlife was not associated with Alzheimer's disease in later life.

\section{The role of blood pressure}

Two previous longitudinal, population based studies have shown an association between raised blood pressure and subsequent Alzheimer's disease. ${ }^{12}$ These studies, however, suggested that the risk was related to raised diastolic blood pressure rather than raised systolic pressure. Differences in the study settings and

Table 3 Relation of medical history and vascular characteristics to Alzheimer's disease. Values are numbers (percentages) unless otherwise stated

\begin{tabular}{|c|c|c|c|}
\hline Characteristic* & Alzheimer's disease $(n=48)$ & No dementia ( $n=1352)$ & $P$ valuet \\
\hline \multicolumn{4}{|c|}{ Myocardial infarction: } \\
\hline Midlife & $3(6)$ & $34(2.5)$ & 0.245 \\
\hline Re-examination & $18(38)$ & $189(14.0)$ & $<0.001$ \\
\hline \multicolumn{4}{|c|}{ Cerebrovascular symptoms: } \\
\hline Midlife & $0(0)$ & $19(1.4)$ & 1.00 \\
\hline Re-examination & $9(19)$ & $89(6.6)$ & 0.004 \\
\hline \multicolumn{4}{|l|}{ Diabetes mellitus: } \\
\hline Midlife & $0(0)$ & $16(1.2)$ & 0.929 \\
\hline Re-examination & $6(13)$ & $88(6.5)$ & 0.177 \\
\hline \multicolumn{4}{|c|}{ Antihypertensive drug treatment: } \\
\hline Midlife & $13(27)$ & $194(14.3)$ & 0.015 \\
\hline Re-examination & $19(40)$ & $488(36.1)$ & 0.621 \\
\hline \multicolumn{4}{|c|}{ Cholesterol lowering drug treatment: } \\
\hline Re-examination & $10(21)$ & $208(15.4)$ & 0.436 \\
\hline \multicolumn{4}{|l|}{ Smoking: } \\
\hline Midlife & $23(48)$ & $577(42.7)$ & 0.474 \\
\hline Re-examination & $19(40)$ & $498(36.8)$ & 0.929 \\
\hline \multicolumn{4}{|l|}{ Alcohol use: } \\
\hline Midlife & $29(60)$ & $956(70.7)$ & 0.633 \\
\hline Re-examination & $22(46)$ & $964(71.3)$ & 0.003 \\
\hline
\end{tabular}

*Information was obtained from detailed questionnaires about medical history and medical conditions and events diagnosed by a physician. Midlife and re-examination indicates the visit when the questionnaire was answered.

$\dagger \chi^{2}$ test was used. 
populations could account for the discrepancy. For instance, treatment patterns may have contributed to our finding. Participants with Alzheimer's disease were more likely to have received antihypertensive drug treatment in midlife but, despite the treatment, still had higher systolic blood pressure in midlife than their counterparts without dementia. This is in line with findings that a considerable proportion of treated hypertensive patients do not achieve the target blood pressure and the fact that diastolic blood pressure has traditionally been the main indication for antihypertensive treatment. ${ }^{2} 10$

Our study, including both treated and untreated participants, may underestimate the risk related to diastolic blood pressure. Notably, in JapaneseAmerican men the raised diastolic blood pressure in midlife predicted Alzheimer's disease only in participants never treated with antihypertensive drugs. ${ }^{2}$ From this perspective, our data should not be interpreted as discounting the potential risk of Alzheimer's disease related to raised diastolic blood pressure but rather as emphasising the importance of raised systolic blood pressure, even in people with normal diastolic blood pressure. Our findings are partly corroborated by the only drug trial to date showing that blood pressure control may prevent dementia, which was carried out in patients with isolated systolic hypertension. ${ }^{11}$

High serum total cholesterol concentration in midlife also predicted Alzheimer's disease in later life. This finding is consistent with the findings in elderly Finnish men.. ${ }^{3}$ Our study extends these findings to younger age groups and both sexes, making these data more representative.

\section{The role of cholesterol}

In this study, clinical indicators of atherosclerosis were more common in patients with Alzheimer's disease than in participants without dementia. A population based, cross sectional study has previously indicated an increased risk of Alzheimer's disease in patients with atherosclerosis. ${ }^{12}$ Hypertension and hypercholesterolaemia may increase the risk of dementia by inducing atherosclerosis and impairing blood flow, but they may also directly induce the neurodegeneration of Alzheimer's disease. ${ }^{13}$ Controlling for vascular events did not change the association between midlife high systolic blood pressure and cholesterol concentration and subsequent Alzheimer's disease, suggesting that hypertension and hypercholesterolaemia themselves pose a risk for Alzheimer's disease. Furthermore, the combination of hypertension and hypercholesterolaemia in midlife was a particularly strong predictor of Alzheimer's disease; these factors may accelerate the development of Alzheimer's disease partly through different pathophysiological mechanisms.

Apolipoprotein $\mathrm{E}$ genotype may influence the observed associations, but more than $85 \%$ of the variation in serum cholesterol concentrations is thought to be independent of the apolipoprotein $\mathrm{E}$ genotype. ${ }^{14}$ Accordingly, the findings in elderly Finnish men suggested that high serum cholesterol concentration was an independent risk factor for Alzheimer's disease regardless of the apolipoprotein $\mathrm{E}$ genotype. ${ }^{3}$ However, more research is needed to determine if apolipoprotein E genotype modulates the effects of risk factors for Alzheimer's disease.

\section{What is already known on this topic}

Vascular risk factors may play an important part as risk factors for Alzheimer's disease

No population based studies have evaluated prospectively the impact of both midlife blood pressure and cholesterol concentration in both men and women on the subsequent development of Alzheimer's disease

\section{What this study adds}

Raised systolic blood pressure and high serum cholesterol concentration, and in particular the combination of these risks, in midlife increased the risk of Alzheimer's disease in later life

Raised systolic blood pressure and hypercholesterolaemia may have a role in the pathogenesis of Alzheimer's disease; more emphasis should be placed on identification and appropriate treatment of these conditions

\section{Study methodology}

The design of our study as a population based, longitudinal study with a large cohort of participants and substantial response rate increases the credibility of these findings. No autopsy data were available to confirm the clinical diagnosis, although the accuracy of the clinical diagnosis of Alzheimer's disease at Kuopio University Hospital, verified by neuropathology, has been reported to be $96 \%{ }^{15}$

We investigated the possibility of selection bias due to non-participation. The prevalences of both raised systolic blood pressure and hypercholesterolaemia in midlife were higher among non-participants. People with cognitive decline are less likely to participate in clinical studies. ${ }^{16}$ Hence, if non-participants were at an increased risk of cognitive impairment and dementia, our results would represent an underestimate of the true effect of the risk factors rather than the opposite. Only participants scoring $\leqslant 24$ in the mini-mental state examination in the screening phase underwent the exhaustive examinations needed for the diagnosis of dementia. Some dementia cases may have been lost because of this cut-off score, and this may also have resulted in underestimation of the prevalence of dementia.

\section{Conclusion}

As the proportion of elderly people in the population increases, Alzheimer's disease will become an enormous public health problem. Interventions that could delay the onset of the disease, even modestly, would therefore have a major impact on public health. ${ }^{17}$ The observed relation between midlife vascular risk factors and Alzheimer's disease later in life may have implications for the prevention of dementia as both hypertension and hypercholesterolaemia can be treated.

We thank Veli Koistinen, Veikko Jokela, and Pirjo Halonen for statistical help and Liisi Saarela for technical assistance.

Contributors: MK and E-LH were the principal investigators. MK analysed the data and drafted the paper. E-LH assisted in analyses and writing. E-LH, AN, JT, and HS contributed to the conception and design of the study, and $\mathrm{AN}$ and JT were also 
involved in the baseline surveys for the study. MH and KA participated in the diagnosis of dementia. MPL and TH participated in analysing the data and writing the paper. All the authors took part in planning the study and interpreting the data and commented on the manuscript. MK is the guarantor.

Funding: Academy of Finland grants 37573 and 63645 and EVO grant 477268.

Competing interests: None declared.

1 Skoog I, Lernfelt B, Landahl S, Palmertz B, Andreasson L-A, Nilsson L, et al. 15-year longitudinal study of blood pressure and dementia. Lance 1996:347:1141-5.

Launer LJ, Ross GW, Petrovitch H, Masaki K, Foley D, White LR, et al. Midlife blood pressure and dementia: the Honolulu-Asia aging study. Neurobiol Aging 2000;21:49-55.

3 Notkola I-L, Sulkava R, Pekkanen J, Erkinjuntti T, Ehnholm C, Kivinen P, et al. Serum total cholesterol, apolipoprotein E4, and Alzheimer's disease. Neuroepidemiology 1998:17:14-20.

4 Braak E, Griffing K, Arai K, Bohl J, Bratzke H, Braak H. Neuropathology of Alzheimer's disease: what is new since A. Alzheimer? Eur Arch Psychiatry Clin Neurosci 1999;249(suppl 3):14-22.

5 Vartiainen E, Puska P, Jousilahti P, Korhonen HJ, Tuomilehto J, Nissinen A. Twenty-year trends in coronary risk factors in North-Karelia and in other areas of Finland. Int J Epidemiol 1994;23:495-504.

6 American Psychiatric Association. Diagnostic and statistical manual of men tal disorders. 4th ed. Washington DC: American Psychiatric Association, 1994.

7 McKhann G, Drachman D, Folstein M, Katzman R, Price D, Stadlan EM. Clinical diagnosis of Alzheimer's disease: report of the NINCDS-ADRDA work group under the auspices of Department of Health and Human Services Task Force on Alzheimer's Disease. Neurology 1984;34:939-44.

8 Hachinski VC, Iliff LD, Zilhka E, Du Boulay GH, McAllister VL, Marshall J, et al. Cerebral blood flow in dementia. Arch Neurol 1975;32:632-7.

9 Klungel OF, Kaplan RC, Heckbert SR, Smith NL, Lamaitre RN, Longstreth WT Jr, et al. Control of blood pressure and risk of stroke among pharmacologically treated hypertensive patients. Stroke among phar $000 ; 31: 420-4$.

10 Applegate WB. Hypertension in elderly patients. Ann Intern Med 1989;110:901-15

11 Forette F, Seux M-L, Staessen JA, Thijs L, Birkenhäger WH, Babarskiene $\mathrm{M}-\mathrm{R}$, et al. Prevention of dementia in randomised double-blind placebocontrolled systolic hypertension in Europe (Syst-Eur) trial. Lancet 1998:352:1347-51

12 Hofman A, Ott A, Breteler MMB, Bots ML, Slooter AJ, van Harskamp F, et al. Atherosclerosis, apolipoprotein $\mathrm{E}$, and prevalence of dementia and Alzheimer's disease in the Rotterdam study. Lancet 1997;349:151-4

13 Skoog I, Kalaria RN, Breteler MMB. Vascular factors and Alzheimer disease. Alzheimer Dis Assoc Disord 1999;13(suppl 3):106-14.

14 Davignon J, Gregg RE, Sing CF. Apolipoprotein E polymorphism and atherosclerosis. Arteriosclerosis 1988;8:1-21.

15 Kosunen O, Soininen H, Paljärvi L, Heinonen O, Talasniemi S, Riekkinen PJ Sr. Diagnostic accuracy of Alzheimer's disease: a neuropathological study. Acta Neuropathol 1996;91:185-93

16 Launer LJ, Wind WA, Deeg DJH. Nonresponse pattern and bias in a community-based cross-sectional study of cognitive functioning among the elderly. Am J Epidemiol 1994;139:803-12.

17 Brookmeyer R, Gray S, Kawas C. Projections of Alzheimer's disease in the United States and the public health impact of delaying disease onset. $\mathrm{Am}$ J Public Health 1998;88:1337-49.

(Accepted 19 March 2001)

\section{A cognitive behavioural intervention to reduce sexually transmitted infections among gay men: randomised trial}

John Imrie, Judith M Stephenson, Frances M Cowan, Shamil Wanigaratne, Andrew J P Billington, Andrew J Copas, Lesley French, Patrick D French, Anne M Johnson for the Behavioural Intervention in Gay Men Project Study Group

\begin{abstract}
Objective To determine the effectiveness of a brief cognitive behavioural intervention in reducing the incidence of sexually transmitted infections among gay men.

Design Randomised controlled trial with 12 months' follow up.

Setting Sexual health clinic in London.

Participants 343 gay men with an acute sexually transmitted infection or who reported having had unprotected anal intercourse in the past year.

Main outcome measures Number of new sexually transmitted infections diagnosed during follow up and self reported incidence of unprotected anal intercourse.

Results 72\% (361/499) of men invited to enter the study did so. $90 \%(308 / 343)$ of participants returned at least one follow up questionnaire or re-attended the clinic and requested a check up for sexually transmitted infections during follow up. At baseline, $37 \%(63 / 172)$ of the intervention group and 30\% $(50 / 166)$ of the control group reported having had unprotected anal intercourse in the past month. At 12 months, the proportions were $27 \%(31 / 114)$ and $32 \%$ (39/124) respectively $(\mathrm{P}=0.56)$. However, $31 \%$ (38/123) of the intervention group and 21\% (35/168) of controls had had at least one new infection diagnosed at the clinic (adjusted odds ratio 1.66, 95\% confidence interval 1.00 to 2.74). Considering only
\end{abstract}

men who requested a check up for sexually transmitted infections, the proportion diagnosed with a new infection was $58 \%(53 / 91)$ for men in the intervention group and $43 \%$ (35/81) for men in the control group (adjusted odds ratio 1.84, 0.99 to 3.40). Using a regional database that includes information from 23 sexual health clinics in London, we determined that few participants had attended other sexual health clinics.

Conclusions This behavioural intervention was acceptable and feasible to deliver, but it did not reduce the risk of acquiring a new sexually transmitted infection among these gay men at high risk. Even carefully designed interventions should not be assumed to bring benefit. It is important to evaluate their effects in randomised trials with objective clinical end points.

\section{Introduction}

Each year in the United Kingdom about 1400 homosexual men are newly diagnosed as being infected with HIV; this figure has remained comparatively stable for a decade. ${ }^{1}$ Gay men attending sexual health clinics in London report engaging in significantly more high risk sexual behaviour than gay men surveyed in community settings. ${ }^{2}$ Effective behavioural interventions to reduce sexual risk taking and infection with HIV and other sexually transmitted diseases are clearly needed, ${ }^{3}$ and sexual health clinics are an appro-
Department of Sexually Transmitted Diseases, Royal Free and University College Medical School, London WC1E 6AU

John Imrie senior research fellow Judith M Stephenson senior lecturer in epidemiology Frances M Cowan senior lecturer in genitourinary medicine

Andrew J Copas lecturer in statistics Anne M Johnson professor of epidemiology continued over

BMJ 2001;322:1451-6

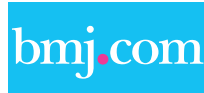

A description of the cognitive behavioural intervention appears on the BMJ's website 